\title{
TTR
}

Traduction, terminologie, re?daction

\section{Relations intersubjectives et marqueurs culturels dans les publicités anglaises et françaises}

\section{Geneviève Quillard}

Volume 11, numéro 1, 1er semestre 1998

Diachronie et synchronie

Diachronics and Synchronics

URI : https://id.erudit.org/iderudit/037319ar

DOI : https://doi.org/10.7202/037319ar

Aller au sommaire du numéro

Éditeur(s)

Association canadienne de traductologie

ISSN

0835-8443 (imprimé)

1708-2188 (numérique)

Découvrir la revue

Citer cet article

Quillard, G. (1998). Relations intersubjectives et marqueurs culturels dans les publicités anglaises et françaises. TTR, 11(1), 137-155.

https://doi.org/10.7202/037319ar
Résumé de l'article

" Relations intersubjectives et marqueurs culturels dans les publicités anglaises et françaises " - Cette étude est basée sur un corpus comprenant d'une part des publicités nord-américaines de langue anglaise et leur traduction en français, d'autre part des publicités publiées en France. Elle se propose de montrer que les relations inter-énonciateurs sont aussi marquées dans les publicités françaises que dans les publicités anglaises. Elle se penchera également sur les rapports que l'émetteur établit avec le destinataire et la façon dont il lui présente son produit, ainsi que sur les différences culturelles que présentent un certain nombre de traductions.
Tous droits réservés (C) TTR: traduction, terminologie, rédaction — Les auteurs, 1998
Cedocument est protégé par la loi sur le droit d'auteur. L'utilisation des services d'Érudit (y compris la reproduction) est assujettie à sa politique d'utilisation que vous pouvez consulter en ligne.

https://apropos.erudit.org/fr/usagers/politique-dutilisation/ 


\section{Relations intersubjectives et marqueurs culturels dans les publicités anglaises et françaises}

\section{Geneviève Quillard}

\section{Introduction}

L'une des idées les plus profondément ancrées en traduction est que, contrairement à l'anglais, le français a tendance * à ne pas marquer la relation inter-énonciateurs " (Guillemin-Flesher, 1986, p. 65). Or, plus $\mathrm{j}$ 'analysais des traductions, plus il me paraissait qu'il s'agissait là d'une affirmation beaucoup trop catégorique, et que, si écart il y avait entre les pratiques discursives des francophones et des anglophones, celui-ci n'était pas le gouffre que l'on nous présentait avec force exemples convaincants.

Pour tenter de vérifier cette hypothèse, j'ai constitué un corpus d'annonces publicitaires. Les publicités sont $\alpha$ exemplaires $\approx$ dans la mesure où elles sont le domaine privilégié des relations intersubjectives, puisqu'elles constituent une forme de " communication motivante * (Albou, 1977, p. 16). Mais, si l'on part du principe que les relations intersubjectives ne sont pas, ou très rarement, marquées en français, on devrait trouver une nette différence même dans ce type de corpus.

Par ailleurs, comme la publicité est un * système d'action qui [...] s'inscrit dans une praxis sociale "(Albou, 1977, p. 201), et que son 
efficacité dépend fortement de la reproduction d'un ensemble de normes et de valeurs partagées par le public auquel elle s'adresse, l'étude des stratégies discursives des publicitaires est susceptible de mettre en lumière certaines différences de comportement entre les anglophones et les francophones.

Ce travail se propose d'analyser les marqueurs de relations interénonciateurs (formes impératives, marqueurs de personne, formes interrogatives), les rapports que l'émetteur établit avec le destinataire et la façon dont il lui présente son produit, ainsi que certaines différences culturelles véhiculées par les changements de point de vue que présentent un certain nombre de traductions.

\section{Corpus et méthodologie}

Le corpus de ce travail comprend :

- 163 publicités extraites de revues nord-américaines de langue anglaise (revues d'actualité, telles que Time Magazine, McLean's, et revues s'adressant à un public féminin, telles que Chatelaine ou Elle Québec) et les traductions de ces 163 publicités parues dans des revues canadiennes de langue française, notamment $L$ 'Actualité et Chatelaine.

- 100 publicités extraites de revues françaises (notamment Le Nouvel Observateur, Le Point, L'Express, Le Figaro Magazine, pour les revues d'actualité et Elle, Marie-Claire, Le Figaro Madame, pour les revues qualifiées de féminines).

Les documents traduits forment évidemment un corpus homogène : même produit, même illustration, même texte. Idéalement il aurait fallu que chaque publicité du corpus de contrôle soit vraiment le pendant d'une publicité de langue anglaise, c'est-à-dire qu'elle porte sur le même produit, la même compagnie ou le même service. Malheureusement, il est difficile d'atteindre cet idéal pour diverses raisons : certains produits se vendent en Amérique du Nord mais pas en Europe, et vice-versa; je disposais également d'un éventail de publicités beaucoup moins large, puisque les revues françaises qui traversent l'Atlantique nous parviennent très mutilées : la plupart des pages 
publicitaires ne quittent pas l'Hexagone. Dans la mesure du possible, j'ai essayé de trouver des publicités correspondantes, c'est-à-dire portant sur le même type de produit ou de service : automobiles, banques, ordinateurs, logiciels, téléphones, compagnies aériennes, compagnies de gaz et d'électricité, appareils électro-ménagers, cosmétiques, produits d'entretien, etc., mais il n'y a pas équivalence à $100 \%$ entre le corpus de publicités nord-américaines et les textes de contrôle.

J'ai commencé par repérer les marqueurs des relations interénonciateurs, qu'ils soient lexicaux (pronoms personnels, pronoms et adjectifs possessifs) ou syntaxiques (interrogations, impératifs)' ${ }^{\prime}$ Toutes les publicités ayant été saisies sur ordinateur, le repérage des marqueurs de personne ou des interrogations, ainsi que le calcul du nombre de mots, ont été effectués par des programmes informatisés. Mais comme l'informatique n'est pas encore la panacée, il a fallu opérer " manuellement " certaines modifications et classifications.

- Pour éviter de fausser les résultats, les pronoms vous et nous des formes pronominales ont été comptés une fois, et non deux; selon cette façon de procéder, le slogan d'une publicité d'Audi, Puisque vous n'aimez pas être en retard nous nous sommes permis de prendre une certaine avance, ne comporte qu'une occurrence du nous. De plus, dans les traductions, les formes pronominales n'ont pas été comptées quand elles ne correspondaient pas a une forme pronominale dans le textesource, comme c'est le cas dans une publicité de Samsonite : You can't afford to travel without us / Vous ne pouvez pas vous permettre de partir en voyage sans nous. En revanche sont comptées toutes les occurrences de you, bien qu'jl ait parfois une valeur impersonnelle.

- Dans un petit nombre de cas est inclus dans les marqueurs de deuxième ou de première personne le pronom dit impersonnel on. Pourquoi? Parce qu'il peut avoir la même portée générale

1 Je n'ai pas analysé les exclamations, car elles ne me semblent pas vraiment constituer des marqueurs de personne. Je ne crois pas, comme J. Delisle (1993, p. 421), que * I'exclamation s'apparente beaucoup a la fausse question *. Au contraire, l'interrogation est centrée sur le destinataire et a donc une fonction expressive, pour reprendre la terminologie de Jakobson, tandis que l'exclamation est centrée sur l'émetteur. 
qu'un you ou la même valeur personnelle qu'un we, comme c'est le cas dans le slogan de la Canadian Imperial Bank of Commerce : We see what you see / On voit la vie de votre façon, de Fujicolor: It's the best you can ask for / On ne peut pas demander mieux, ou dans celui de Total : On ne s'appelle pas Total par hasard.

- La catégorie intitulée " nous général * ( nous $G$ * dans le graphique " variables étudiées ") comprend toutes les formes lexicales qui incluent émetteur et récepteur, telles que : Every so often, life hands us a memory / La vie nous fait parfois cadeau d'un beau sotvenir. (Fujifilm)

- La catégorie " nous compagnie * (" nous $\mathrm{C}$ ") comprend uniquement les formes qui se réfêrent à la compagnie : And our Privacy Code gives consumers / Et notre code de discrétion (Association canadienne du marketing direct).

- Les impératifs n'ont pu être relevés par un programme informatisé. Le risque d'erreur est donc plus élevé. Lorsque la forme impérative apparaissait à plusieurs reprises dans des énoncés tels que : write to us, call us or fax us, elle n'a été comptée qu'une fois.

- La catégorie je est celle de ce que Blanche Grunig (1990, p. 162) appelle le publocuteur, c'est-à-dire de la personne qui dit Je, représentée ou non dans l'illustration, et qui prend le relais du publicitaire : "C'est décidé, je montre mes fesses "; se (nous) promet une des utilisatrices de la crème Cellu-lift de Jeanne Piaubert. Promesse tenue? Hélas, il s'agit d'une " voix " off, et l'illustration représente simplement un tube de cette crème qui risque de faire perdre toute pudeur à celles qui l'utilisent... Il arrive qu'il y ait plus d'un publocuteur. Dans ce cas, les nous ont été inclus dans cette catégorie. Étant donné que cette catégorie est très différente des autres, il n'en sera pas tenu compte dans l'analyse qui suit, mais elle mériterait d'être étudiée de près, car elle véhicule nombre de traits culturels spécifiques à chaque communauté. Il faut remarquer également que les chiffres sont nettement plus élevés en français, parce que les publicités du corpus faisaient beaucoup plus souvent appel à des publocuteurs. C'est peut-être une tendance générale des publicités françaises. De ce point de vue, la démarche française semble être beaucoup plus axée sur le destinataire que sur l'émetteur. Le 
publocuteur est en effet un je bien proche du destinataire. Ce pourrait être elle ou lui, son mari, sa femme, ses enfants, ses parents. C'est donc en quelque sorte lui qui prend la parole, alors que dans les publicités anglaises " Je est un autre ".

Le premier tableau présente le nombre d'occurrences des marqueurs étudiés, le second le pourcentage de ces marqueurs par rapport au nombre de mots. Le troisième présente les données du second tableau sous forme de graphique.

\begin{tabular}{|l|l|l|l|}
\hline & anglais & traduction & français \\
\hline$n^{\text {o de publicités }}$ & 163 & 163 & 100 \\
\hline $\mathrm{n}^{\text {o de mots }}$ & 26139 & 29854 & 13069 \\
\hline impératifs & 255 & 211 & 60 \\
\hline nous compagnie & 266 & 284 & 93 \\
\hline nous général & 15 & 29 & 44 \\
\hline vous & 816 & 774 & 539 \\
\hline questions & 45 & 64 & 72 \\
\hline je & 30 & 24 & 53 \\
\hline
\end{tabular}




\begin{tabular}{|l|l|l|l|}
\hline & anglais & traduction & français \\
\hline impératifs & $0.98 \%$ & $0.71 \%$ & $0.46 \%$ \\
\hline nous compagnie & $1.02 \%$ & $0.95 \%$ & $0.71 \%$ \\
\hline nous général & $0.06 \%$ & $0.10 \%$ & $0.34 \%$ \\
\hline vous & $3.12 \%$ & $2.59 \%$ & $4.12 \%$ \\
\hline questions & $0.17 \%$ & $0.21 \%$ & $0.55 \%$ \\
\hline je & $0.11 \%$ & $0.08 \%$ & $0.41 \%$ \\
\hline
\end{tabular}

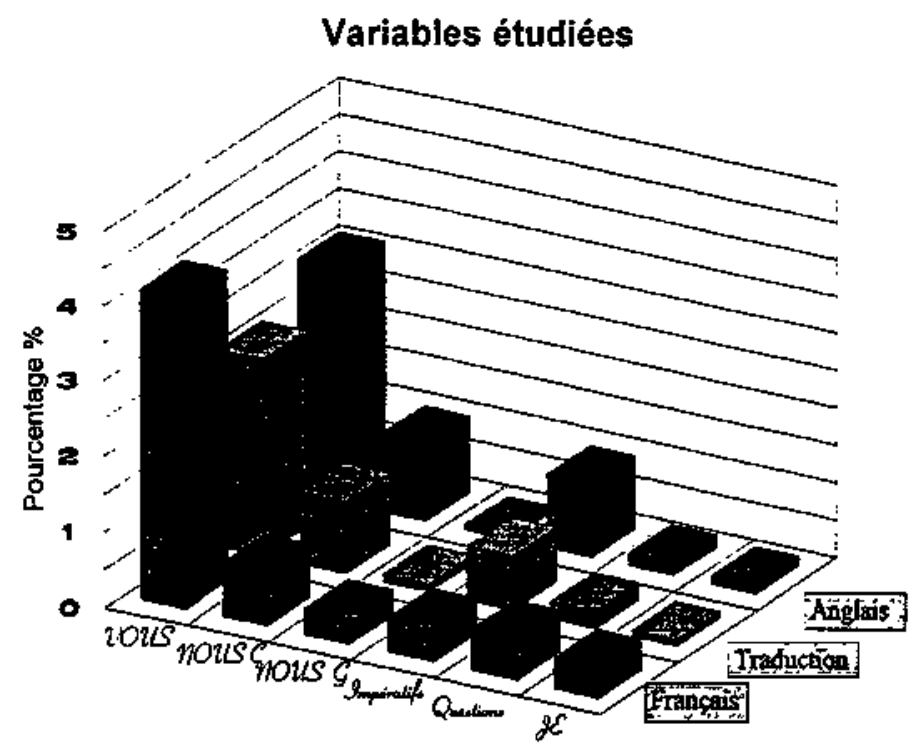




\section{Marqueurs de la relation intersubjective}

\section{Impératifs}

Les données obtenues dans le corpus de traductions montrent que les traducteurs n'ont pas hésité à occire un certain nombre de formes impératives; il en survit tout de même beaucoup plus que dans les textes de contrôle. La différence entre l'anglais et le français est en effet astronomique, puisque les impératifs sont deux fois plus nombreux dans les publicités anglaises. Ces chiffres indiquent clairement que les francophones ne prisent guère les actes de langage directifs ${ }^{2}$, qui établissent entre les interlocuteurs des rapports hiérarchisés et dissymétriques. Cela est particulièrement clair à la fin des annonces. Alors qu'en anglais elles se terminent pratiquement toutes par : call us, fox us, come and see us, visit your nearest agent, quand ce n'est pas sous une forme encore plus insistante : call us today, en France on se contente généralement de donner le numéro de téléphone, de télécopieur ou le code de Minitel.

Une publicité de la compagnie aérienne Delta montre bien comment les impératifs sont de plus en plus décimés quand ils passent dans les rangs canadiens français et français :

Delta/see

suit / consultez

- anglais : 88 mots, 3 impératifs : consider this / fly

- CF : 92 mots, 2 impératifs : lisez attentivement ce qui

- FF : 98 mots, 1 impératif : contactez.

L'impératif mériterait une étude approfondie sur le plan sémantique pour déterminer s'il possède une valeur personnelle ou impersonnelle et quel acte de langage il exprime (injonction, interdiction, requête, suggestion, invitation, conseil...). Mais, quel que soit l'acte de langage, l'impératif est une forme directe, tout comme le should, qui

${ }^{2} \propto$ En publicité le consommateur québécois n'aime pas les impératifs du genre - Buy it now! * Cette formule si chère aux Américains ne laisse pas le temps mental de finasser avec l'annonceur et bloque l'action recherchée. "(Bouchard, 1978, p. 79) 
mériterait lui aussi d'être étudié de près. Le français lui préfêre nettement les formes directives indirectes ou « déguisées $*: A$ vous maintenant de les découvrir (Peugeot 405); Et si vous vous mettiez à la vitalité? (Vittel); call Unisys / vous n'avez plus qu'à appeler Unisys; Simply SkyPak it / SkyPak, tout simplement. Il est interressant de noter que la forme infinitive ne paraît qu'une seule fois dans ce corpus ${ }^{3}$.

Enfin, dans le corpus de contrôle, les formes impératives peuvent inclure aussi bien le destinateur que le destinataire, puisqu'elles sont parfois à la première personne, ce qui est rarissime dans les textes anglais.

Nous compagnie. Les occurrences du marqueur de première personne présentent aussi une très nette différence entre le corpus anglais et le corpus français, et une légère différence entre le corpus anglais et le corpus de traductions. Divers facteurs expliquent ces différences.

1. Les publicitaires anglophones se mettent fréquemment de l'avant pour vanter les mérites de leur marchandise, alors qu'en France ils tendent à s'effacer derrière leur produit. C'est peut-être ce qui explique que les publicités soient souvent beaucoup plus courtes en France. ${ }^{4}$

2. Les assertions sont beaucoup plus souvent modalisces en anglais qu'en français. Toutefois ces modalisations ne sont pas toujours un moyen d'atténuer l'assertion, comme c'est le cas dans : We think you'll agree that it will give you a considerable advantage over your competition / Bel avantage sur vos concurrents, n'est-ce-pas? (Grid), ou We think you'll be, at least, mildly impressed / il a de quoi vous

${ }^{3}$ À en juger par ce qu'écrit Dupont (1993, p. 92), l'infinitif est une forme peu recommandable en rédaction publicitaire : * C'est le mode le plus impersonnel qui soit. Des formules telles que " L'essayer c'est l'adopter o ne font plaisir qu'a l'orgueil de l'annonceur. "

${ }^{4}$ Dans le corpus qui fait l'objet de cette étude, du moins. Toutefois une étude des titres semble confirmer ceci : « Analysant 330 titres français et 260 titres américains, Claude Raymond Haas a remarqué que la moyenne des mots par titre s'établit à 5,32 chez les Français et à 6,62 chez les Américains. * (Dupont, 1993, p. 70) 
impressionner en douceur! (Dove). Elles peuvent être au contraire un moyen de renforcer l'assertion : At Hitachi we know you want the best / Vous êtes exigeant.

3. Les redondances sont pratiquement inexistantes dans le corpus de contrôle, alors qu'elles sont nombreuses dans les textes anglais : at Unysis we believe / Unisys élabore. Elles sont également nettement moins fréquentes dans les traductions. Comment expliquer alors que le nombre de marqueurs de première personne soit un peu plus élevé dans les traductions? Dans la plupart des textes anglais, le nom de la compagnie ou du service revient à intervalles plus ou moins réguliers. L'utilisation du nous permet aux traducteurs d'éviter un certain nombre de répétitions.

Nous général. La différence de fréquence de ce marqueur dans les trois groupes semble, elle aussi, assez révélatrice. Les textes français et les traductions établissent des rapports d'inclusion entre les participants de l'acte de communication, alors que le destinataire est nettement moins souvent interpellé dans les textes anglais.

Vous. Ils sont beaucoup moins nombreux dans les traductions que dans les textes de contrôle. Cela vient peut-être de ce que les traducteurs ont bien appris leur leçon : il faut à tout prix dépersonnaliser le message, évincer le destinataire du texte. Sus aux marqueurs de deuxième personne! En revanche, leur nombre est plus élevé en français qu'en anglais. Certes, il faudrait un corpus beaucoup plus important que celui-ci pour qu'on puisse totalement se fier aux statistiques. Celles-ci n'ont qu'une valeur indicative. Elles donnent tout de même à penser que l'inscription du destinataire dans les textes français n'est pas un phénomène aussi rare qu'on l'avait cru jusqu'ici.

Questions. La fréquence de cet acte de langage est très inégale dans les deux langues, comme elle l'est d'ailleurs dans beaucoup de communautés linguistiques (Kerbrat-Orecchioni, 1991, p. 37). Ces résultats confirment ceux d'une étude antérieure (Pons-Ridler, Quillard, 1993). Après avoir dépouillé trois revues de langue anglaise et trois revues de langue française, nous avions trouvé presque deux fois plus de questions en français qu'en anglais. Or la question est à l'impératif ce que la médecine douce est aux traitements de choc : elle permet d'inscrire le destinataire 
en douceur dans le texte, c'est-à-dire sans risque de le heurter ou de le choquer : Keep your Windows 95 software in one of our beautiful, customdesigned cases / Pourquoi ne pas ranger votre système d'exploitation Windows 95 dans l'un de nos superbes coffrets fabriqués sur mesure? (Digital). Il me semble également qu'elle donne au texte écrit une perspective dialogale et interactive beaucoup plus vivante et peut-être aussi plus proche de la réalité, dans la mesure où les interrogations forment une partie intégrante des échanges en face à face ${ }^{5}$.

\section{Rapports inter-énonciateurs}

\section{Présentation de soi et rapports entretenus avec le destinataire}

Si, comme l'écrit C. Kerbrat-Orecchioni : « la loi de modestie * [...] s'exerce dans nos sociétés elles-mêmes de façon passablement tyrannique " (1992, p. 186), elle paraît tout de même tyranniser beaucoup moins les anglophones que les francophones. D'après une des maximes de modestie « l'on ne doit pas se glorifier soi-même. [...] À la rigueur, si l'on est amené à faire son propre éloge, on peut et doit user de la litote, ou autre précaution " ravalante ". " (p. 186) Cette maxime ne semble pas être très suivie par les publicitaires anglophones, qui n'hésitent pas à exprimer leur auto-satisfaction sans aucun détour et sans aucune précaution oratoire. Dans la plupart des cas, le mutisme des traductions est révélateur. Le francophone éprouve beaucoup de mal à se féliciter luimême de ses exploits :

An engine so advanced that it could only have come from the engineers at $B M W / \ldots$

Inspiration is commonplace in the Jetta $/ \ldots$

Everything is as it should be. /... (KLM)

\footnotetext{
${ }^{5}$ Parmi les 25 principes élémentaires que, selon Dupont (1993, p. 89), doivent respecter ceux qui veulent " écrire des textes qui vendent ", figure celui-ci : * Utilisez des phrases personnelles. Sont définies comme phrases personnelles, les phrases écrites sur les modes de la conversation. "(En italiques dans le texte.)
} 
And nowhere do we realize our efforts more successfully /... (Toshiba)

The most advanced we've ever built /... (Hitachi)

Les rapports entretenus avec le destinataire sont parfois eux aussi de nature à heurter la sensibilité des francophones : on les traite un peu de haut, on se met a leur place ou on leur donne des ordres. Le slogan d'Hitachi est exemplaire à cet égard : Demand it / Rien de moins. Les traducteurs modifient alors le texte avec beaucoup de doigté pour que la * face " du destinataire ne soit plus menacée ou que disparaissent les présupposés potentiellement insultants pour un francophone, comme dans le quatrieme exemple, où le texte français ne présuppose pas que le destinataire suit une thérapie (il s'agit probablement ici d'une différence d'ordre culturel) :

What you probably don't know / Mais saviez-vous que (Sears, KidVantage)

-So, just how big is the pickup you're driving right now? - Well? / Avezvous une idée à quel point notre camionnette 2 ou 4 roues motrices est grande? (Toyota T100)

Reflecting the level of thinking and quality that you've come to expect from Compaq / C'est le reflet de la nouvelle pensée et de l'engagement envers la qualité qui tiennent tant à coeur à Compaq

So the next person you can fire is your therapist / Ainsi, vous pourrez aussi vous passer d'un thérapeute (Compaq)

And do yourself a favour, don't just take our word for it. / Et surtout. $n$ 'allez pas nous croire sur parole (Compaq)

when it comes to performance you should demand the best /... (Hitachi)

\section{Présentation du produit}

De même que les publicitaires anglophones se présentent généralement sous un jour très favorable, ils vantent les qualités de leur produit avec force superlatifs et hyperboles : these diverse and brilliant machines, the stunning technology... without peer in the universe of colour (Canon); newly advanced filtering lenses / lentilles filtrantes (Hitachi). Les 
publicités françaises ne sont pas toutes purement descriptives, mais elles sont nettement plus modérées. On trouve ici et là des évaluations qualitatives : une insonorisation parfaite (Peugeot), une ergonomie exceptionnelle (Olivetti), des propriétés uniques (Vittel), de remarquables mécaniques (Renault), mais elles demeurent discrètes et, dans ce corpus, elles n'apparaissent jamais plus d'une fois dans un même texte. En ce qui concerne les textes cibles, il est évident que les traducteurs arrivent vite à saturation devant la logorrhée d'adjectifs et d'adverbes mélioratifs. Ils ont alors recours à l'une des trois stratégies suivantes.

a. Adoption d'une optique différente, généralement basée sur des données quantitatives plutôt que sur des jugements qualitatifs ou sur des faits plutôt que sur des mythes :

It would even have things like a simple trunk hinge that's the best you have ever seen / Elle serait même dotée de charnières de coffre simplifiées permettant de multipller l'espace de chargement (Honda Accord)

A new legend begins. Introducing Kodak diskettes / Oui, Kodak fait aussi des disquettes. Du nouvean : les disquettes Kodak

b. Amalgame en un seul terme de plusieurs éléments mélioratifs:

The stunning picture. The unbellevable clarity. The spectacular sound / Vous desirez une image, une définition et un son exceptionnels (Hitachi)

c. Élimination des mots ou des phrases qui vantent tellement les avantages du produit que le francophone risque de les mettre en doute et de se demander ce qui se cache derrière l'insistance du publicitaire :

The AM/FM cassette stereo cleverhy adjusts its own volume / Le système $A M / F M$ stéréo d̀ lecteur de cassettes règle son propre volume (Mercedes)

the exciting dermatological alternative /la solution dermatologique (NeoStrata)

an innovative case /... (Samsonite) 
exquisite... superb, the ultimate /... (Clarins)

Just terrific colour. Absolutely beautiful / Des teintes riches et colorées (Marcelle)

a remarkable variety of items from the practical to the sublime /... (Royal Bank Visa Gold)

the Jenn-Air range's sleek, streamlined exterior $/$...

no fewer than eight brilliant new models will be unveiled / BMW lancera pas moins de huit nouveaux modèles

\section{Différences culturelles}

Étant donné que le domaine de la publicité, * c'est l'humain et le social * (Brochand, Lendrevie, 1985, p. 8), elle reproduit un grand nombre de pratiques culturelles (tant dans l'illustration que dans le message, d'ailleurs).

Les pratiques alimentaires et culinaires des anglophones et des francophones n'étant pas tout à fait les mêmes, est-il étonnant que les traductions prennent certaines libertés? Traduit littéralement, le premier exemple n'aurait pas manqué de susciter l'ire des francophones! Quant au most du second exemple, il est irrecevable pour un francophone, qu'il s'agisse ou non d'un mythe. Il est intéressant aussi de trouver un jeu de mots dans la traduction, alors qu'il n'y en a pas dans le texte anglais :

The vintage wine is cool and the meal is hot / Le vin grand cru est parfaitement chambré et le repas est fin (KL,M)

Mast mums know great taste makes the meal / Protvez aux membres de votre famille que vous avez du goüt (Lipton)

Pour ce qui a trait à l'argent, les anglophones en parlent très ouvertement, alors que c'est un sujet que les francophones prefferent éviter :

Ultravalet -- the best garment bag you can buy//Le sac à vêtements Ultravalet n'a pas son pareill (Samsonite)

We spare no expense to make them pure / Nous n'avons rien ménagé pour mettre au point un produit pur (Webber) 
The formula has one of the toughest acne-fighters you can buy / Sa formule est l'une des plus efficaces (Cover Girl)

Certains comportements sociaux ou certaines réalités de la vie ne sont pas traduits en français avec autant de réalisme ou de candeur. Ainsi, dans le premier exemple, la G20 n'est plus là pour susciter l'envie, ce qui donne à penser que Canadiens français et Français ont la même attitude envers ce type de comportement : Les récepteurs français supportent [...] assez mal que dans la publicité - explicitement (l'implicite peut être autre...) - on déprécie une partie de la société par rapport à d'autres n (Grunig, 1990, p. 217). Cette observation rejoint celle de Bouchard : « L'appel direct à l'émulation, si peu québécois, tellement américain, comme dans « Le voisin a une Cadillac, pourquoi pas vous? » ou * Tout le monde en a, pourquoi pas toi " est un argument négatif chez nous ” $\left(1978\right.$, p. 173) ${ }^{6}$ :

The spacious interior that allows you to share the pleasures of your G20 with friends (and perhaps engender a little envy at the same time) / En route, I'habitacle spacieux accueille vas amis qui partageront avec vous les sensations uniques que procure la G20 (Nissan)

Who taught them to eat at the table, use the potty, and ride a bike? I Qui lui a montré à manger et à faire de la bicyclette? (Sears, KidVantage)

Today, it takes ... perspiration / Aujourd 'hui, il faut du travall acharné (Midland Walwyn)

S'il leur arrive d'effectuer des coupures pour éviter d'enfreindre certaines règles de comportement social, les traducteurs rajoutent parfois des éléments au texte-source. Ces éléments sont toujours centrés directement ou indirectement sur le destinataire :

\footnotetext{
${ }^{6}$ Bouchard ajoute : $\propto$ il est quand mème beaucoup utilisé en publicité traduite . Il arrive certes qu'il se produise des « dérapages " comme celui-ci : In the past, North Americans could onty stand and salivate. Now they can buy Isouligne dans le textel them / Jusqu'à présent, les Nord-Américains devaient se contenter de les regarder passer en salivant d'envie. Maintenant, ils peuvent les acheterl (BMW). Mais les traductions de ce corpus se laissent rarement prendre au piège des interférences cuiturelles.
} 


\section{... / $\grave{A}$ votre santé! (jus de raisin Welch)}

Then add lemon, tomato and clam juice / Ajoutez du jus de citron, jus de tomates et palourdes et... dégustez (Beefeater)

... / Le vert vous ira bien! (Canon. Il s'agit d'un copieur écologique, en noir et blanc)

... / Pour avoir la tendresse à fleur de peau (Nivea)

Il est intéressant de remarquer que, dans les traductions, les jeux de mots sont beaucoup plus nombreux que dans les textes-sources. Or quelle est la fonction des jeux de mots? D'attirer l'attention, bien sûr, mais ils instaurent aussi une certaine connivence entre les partenaires de l'échange, tissant entre eux des liens plus étroits. De plus, le recours à l'humour présente un autre avantage à la fois économique et culturel : * La publicité qui reflète l'exubérance [du Québécois] réussit des percées rapides et durables. [...] "Les commerciaux dröles, ça me dérange moins... Quand l'annonce est sérieuse, c'est qu'elle te cache quelque chose" " (Bouchard, 1978, p. 194) :

Taste. We've got more on our side / Votre famille sourira al dente (Lipton)

Until now, black and white copiers hove been just that -- black and white / Jusqu 'à présent, les copieurs noir et blanc manquaient un peu de couleur (Canon)

hoarding information in an important pile on the credenza doesn't make an executive more powerful / être assis sur une pile d'informations $n$ 'est plus considéré par les cadres comme le meilleur moyen d'asseoir ses positions (Microsoft)

Infiniti Nissan ( $G$ a letter of introduction). For, above all, the G20 luxury sports sedan is an Infiniti / Infiniti Nissan ( $G$ une lettre recommandée). La G20 est une Infiniti recommandable sous tous rapports

... / bref, Infiniment Infinitl!

Not Intended for the General Public / La marque qui vous démarque (Jeep eagle) 
We are a different kind of bank/ Nous faisons banque à part (Banque fédérale de développement)

Les évidences disparaissent, ou bien la traduction présente les choses sous un angle différent :

And in the winter, a heater / Et un système de chauffage (Mercedes)

Those businesses may have private offices and walls, but minds are open / Dans ces entreprises les employés ferment parfois la porte de leur bureau, mais cela ne les empêche pas de garder l'esprit ouvert (Microsoft)

Of course, the G60 super-charger nestles under the hood, its power complementing the syncro's handling / Et, bien sur, le surcompresseur G60 est le complèment direct du système synchro (Volkswagen Passat)

Your skin is yours alone, and the only one you will ever have / La peau est quelque chose d'unique (Webber)

You feel like you 've covered all of Europe in three days and two nights IVous avez l'impression d'avoir parcouru toute l'Europe en trois jours (KLM)

Les répétitions sont elles aussi souvent éliminées :

After all, Mercedes are often driven for 15 years /... [il a été noté auparavant que les Mercedes sont conçues pour avoir aussi bonne allure dans quinze ans qu'aujourd'hui]

It is a cream that acts on your skin's past, present and future /... [Cela a déjà été dit dans le sous-slogan : Anti-time day care cream. Past. Present. Future / Soin de jour anti-temps Passé. Présent. Futur] (Vichy)

You can virtually write your own ticket. /... [dẹjà ditt une fois] (Royal Bank Visa Gold)

\section{Conclusion}

Les résultats de cette recherche montrent que, dans le domaine publicitaire du moins, les rapports inter-énonciateurs sont non seulement aussi 
marqués en français qu'en anglais, mais qu'ils le sont même plus. Les comportements relationnels varient toutefois considérablement d'une culture à l'autre. Les anglophones recourent fréquemment à des formes directives, tandis que les francophones font plutôt appel à des formes indirectes. Les publicitaires anglophones se mettent souvent de l'avant, les francophones se montrent plus effacés et établissent avec le destinataire des rapports plus inclusifs. Il serait intéressant d'effectuer des analyses sur d'autres types de textes pour déterminer la fréquence des rapports intersubjectifs en anglais et en français et pour dessiner un profil typologique de ces rapports.

Les données fournies par cette étude confirment les conclusions d'une recherche portant sur des textes pragmatiques (Quillard, 1997), notamment en ce qui concerne la plus grande fréquence en anglais des formes impératives, des évidences et des redondances. Elles confirment également les résultats d'une étude (Quillard, 1995) portant sur 115 publicités anglaises et leurs traductions, et sur 62 publicités françaises, dans laquelle les pourcentages obtenus étaient les suivants :

\begin{tabular}{|l|l|l|l|}
\hline & anglais & traduction & français \\
\hline impératifs & $0.85 \%$ & $0.62 \%$ & $0.37 \%$ \\
\hline vous & $3.16 \%$ & $2.68 \%$ & $3.05 \%$ \\
\hline questions & $0.16 \%$ & $0.23 \%$ & $0.33 \%$ \\
\hline je & $0.17 \%$ & $0.12 \%$ & $0.27 \%$ \\
\hline
\end{tabular}

(Comme, dans cette étude, les occurrences du nous n'avaient pas été divisées en deux catégories, elles ne figurent pas dans ce tableau.) La seule différence notable de ces deux recherches est au niveau du vous, dont les occurrences étaient légèrement moins fréquentes en français qu'en anglais dans le premier corpus, alors qu'elles sont supérieures dans celui-ci.

Ce travail n'a fait qu'effleurer le domaine culturel. Il s'agit là d'un champ presque totalement inexploré et qui devrait se révéler extrêmement riche. Les études comparatives dans le domaine publicitaire 
devraient jeter une lumière considérable sur ce qui différencie, ou ce qui rapproche, les pratiques culturelles des différentes communautés visées par les messages publicitaires. Il serait notamment intéressant de déterminer si les différences entre l'anglais et le français européens sont plus ou moins grandes qu'entre l'anglais et le français nord-américains.

\section{Collège militaire royal du Canada}

\section{Références}

ALBOU, Paul (1977). Psychologie de la vente et de la publicité. Paris, P.U.F.

BOUCHARD, Jacques (1978). Les 36 cordes sensibles des Québécois. Montréal, Éditions Héritage.

BROCHAND, Bernard et Jacques LENDREVIE (1985), Le publicitor. Paris, Dalloz.

DELISLE, Jean (1993). La traduction raisonnée. Ottawa, Presses de l'Université d'Ottawa.

DUPONT, Luc (1993). 1001 trucs publicitaires. Montréal, Éditions Transcontinentales.

GRUNIG, Blanche (1990). Les mots de la publicité. Paris, Presses du CNRS.

GUILLEMIN-FLESCHER, Jacqueline (1986). * Le linguiste devant la traduction w. Fabula, 7, pp. 59-69.

KERBRAT-ORECCHIONI, Catherine (1991). La question. Lyon, Presses Universitaires de Lyon.

- (1992). Les interactions verbales. Tome II. Paris, Armand Colin.

PONS-RIDLER, Suzanne et Genevieve QUILLARD (1993). "L'interrogation dans les textes écrits. Fréquence et fonctions en anglais et en français ». Interface, 8, 1, pp. 43-56. 
QUILLARD, Geneviève (1992). * Quelques aspects linguistiques et culturels de l'implicite et de l'explicite en anglais et en français ". Interface, 6, 1, pp. 83-91.

- (1995). * Les relations inter-énonciateurs dans les publicités anglaises et françaises ". Communication présentée à Montréal, dans le cadre du colloque des Sociétés savantes.

- (1997). “ Étude de certaines différences dans l'organisation collective des discours anglais et français n. Babel, 43,4 .

RÉSUME : « Relations intersubjectives et marqueurs culturels dans les publicités anglaises et françaises $»-$ Cette étude est basée sur un corpus comprenant d'une part des publicités nord-américaines de langue anglaise et leur traduction en français, d'autre part des publicités publiées en France. Elle se propose de montrer que les relations inter-énonciateurs sont aussi marquées dans les publicités françaises que dans les publicités anglaises. Elle se penchera également sur les rapports que l'émetteur établit avec le destinataire et la façon dont il lui présente son produit, ainsi que sur les différences culturelles que présentent un certain nombre de traductions.

ABSTRACT : « Intersubjective Relationships and Cultural Markers in English and French Advertisements " - This paper is based on a corpus consisting, on one hand, of English North American advertisements and their translations in French, and, on the other, of advertisements published in France. Its aim is to show that intersubjective relationships are as present in French advertisements as in English ones. This paper will also look into the relationship that the sender establishes with the receptor and the way in which he presents his product to him, as well as cultural differences presented by a certain number of translations.

Geneviève Quillard : Département d'Études françaises, Collège militaire royal du Canada, CP 17000, Succursale Forces, Kingston (Ontario) K7K 7B4. Courriel : quillard-g@rmc.ca 